\title{
$\angle S$ Research Square \\ Effects of Nutrition Education Supported by Mobile Application on Weight Loss and Quality of Life: A Randomized Controlled Trial
}

\section{Doga Peksever}

Ege University, Institute of Health Sciences, Dept. of Chronical Diseases

\section{Selda Seckiner}

Ege University, Faculty of Medicine

Reci Meseri ( $D$ recimeseri@yahoo.com )

Ege University Faculty of Health Sciences https://orcid.org/0000-0002-2482-3066

\section{Research}

Keywords: obesity, mobile application, nutrition education, quality of life, self-esteem, healthy eating habits

Posted Date: May 29th, 2020

DOl: https://doi.org/10.21203/rs.3.rs-30619/v1

License: (a) (1) This work is licensed under a Creative Commons Attribution 4.0 International License. Read Full License 


\section{Abstract}

Background Obesity is a growing health problem which affects people from all age groups all over the world. Obese people do not feel motivated enough to change their lifestyle behaviors. Mobile applications can be used to motivate people.

Objective To evaluate effect of nutrition education supported by MOtiVE mobile application on weight loss and quality of life (QoL).

Methods In this randomized-controlled study, 79 overweight/obese adult patients who presented to University Hospital Outpatient Clinic between March-September 2018 to consult a dietitian were included. All the participants were provided a weight-loss diet program by the dietitian. Then, participants were randomized to experimental and control groups. During the first interview, all participants completed the questionnaire and anthropometric measurements were done. BMI, the scores obtained from different Quality of Life scales and Healthy Eating Index (HEI) were the dependent variables. The participants in the experimental group were provided with daily messages for 3 months via MOtiVE mobile application designed solely for this study. All the participants were asked to present three months later for a follow-up appointment. Using SPSS 25.0, change in BMI, QoL scores and other variables within both groups was assessed via Wilcoxon signed-rank test and McNemar chi-square test. $p<0.05$ was considered statistically significant.

Results Although 39 cases and 40 controls took the first test, 20 cases and 18 controls participants completed the study. The mean BMI decreased significantly in both groups being more predominant in cases as from $33.8 \pm 6.0 \mathrm{~kg} / \mathrm{m}^{2}$ to $32.8 \pm 5.8 \mathrm{~kg} / \mathrm{m}^{2}(p=0.001)$ in cases and from $33.3 \pm 5.0 \mathrm{~kg} / \mathrm{m}^{2}$ to $32.2 \pm 4.7 \mathrm{~kg} / \mathrm{m}^{2}(p=0.006)$ in controls. Moreover, waist circumference decreased $(p=0.029)$, self-esteem $(p=0.035)$ and healthy eating scores $(p=0.007)$ increased only in cases significantly.

Conclusions Nutrition education supported by MOtiVE mobile application improved anthropometric measurements, self-esteem, quality of life and healthy eating habits of the overweight/obese participants. Free mobile applications can be used in increasing motivation to adopt new behaviors in order to tackle obesity.

Trial Registration: ClinicalTrials.gov NCT04026971

\section{Background}

Obesity is a multifactorial and chronic disease characterized by excessive accumulation of fat in the body resulting from the interaction between genetic and environmental factors. According to World Health Organisation in 2016, more than 1.9 billion adults were overweight and over 650 million were obese(1). In weight loss programs, the most important factor is lifestyle changes. Internet-based programs are potential supporters for individuals participating in weight loss programs because the Internet is easy to access. Studies conducted on this issue have shown that such programs, although 
short-term, lead to positive changes in diet, physical activity level, glycemic control, anthropometric measurements and biochemical parameters(2-5). In several studies, low self-esteem and poor body image were determined to be associated with obesity $(6,7)$. Providing motivational support to achieve lifestyle changes can play a significant role in maintaining weight loss by increasing self-esteem. The main purpose of the present study was to compare the effect of standard education on weight loss, quality of life (QoL) and healthy eating behavior with that of nutrition education supported by mobile application. It was assumed that the mobile applications would increase people's motivation for and adaptation to diet.

\section{Methods}

\section{Trial Design}

The present study were conducted with patients who presented to the University Hospital Endocrine\&Metabolic Diseases Outpatient Clinic between March-September 2018 to consult a dietitian and to receive routine nutrition therapy, aged between 18-64 years and Body Mass Index (BMI) $\geq 25$. The participants were first given the standard nutrition training by the dietitian in the outpatient clinic, and then person-specific diet program. After this, the patients who gave their consent to participate in the study were randomly assigned to the case and control groups. Then, anthropometric measurements of all the participants were performed and the data collection forms were filled in. The "MOtiVE" mobile application for smart phones designed specifically for this study was installed on the smart phones of the cases, and text, visual or video messages were sent to them via this application for three months. The participants in the control group underwent the routine procedure. In line with the routine practice of the outpatient clinic, all the participants were told to present three months later for control appointments. At the end of December 2018, at the end of the three-month follow-up period, the data collection process ended. In a systematic review conducted by Aguilar-Martınez et al, showed that most of the trials last for 2-4 months(8).

Participants and Recruitment Of the patients, those who did not have a smart phone with internet connection, underwent bariatric surgery, took medication or practice a special diet for thyroid problems, diabetes, celiac, gout and kidney diseases and whose body fat analysis was not fulfilled due to the presence of a pacemaker, prosthesis etc. were not included in the study.

According to the results of a study conducted by Allen et al, 25 participants in each group was enough to reach $80 \%$ power(9). In a meta-analysis conducted by Mateo, revealed that many studies had $40-45$ participants in each group, some of them being less(4). When we consider $80 \%$ power with a $\% 5$ error and medium effect size (d:0.5) we had to have 51 participants in each group calculated via G-power. When we evaluate admissions to the outpatient clinic, we saw that each week we can have 4 or 5 new participants. Thus, considering this data, we decided to take 40 participants in each group. The flowchart of the study is given in Figure 1. 
In the present study, randomization was carried out in the following block allocation sequence: $A A B B$, $A B A B, B A B A, B B A A, A B B A, B A A B$.

Change in the BMI and other anthropometic indices and the scores obtained from the Weight Efficacy Lifestyle (WEL) test, Quality of Life Scale SF-36 (QoLSSF-36), Rosenberg Self-Esteem Scale (RSES), Obesity and Weight-Loss Quality of Life Instrument (OWLQoL), Healthy Lifestyle Behavior-II Scale (HLSBII) and Healthy Eating Index (HEI) were the dependent variables.

\section{Measurements:}

\section{Anthropometric measurements}

The body height was measured with a stadiometer whereas the body weight, body fat percentage and BMI were measured with the Tanita BC-418 Segmental Body Composition Analyzer. Waist circumference was measured with a non-elastic standard measuring tape, with the individual wearing light clothes, standing still, in an upright position and with arms open sideward. WC was measured from the midpoint between the distal border of the lowest rib and the superior border of the iliac crest(10). Neck circumference was measured by placing the inelastic measuring tape around the neck from the point just below the laryngeal prominence.

QLSSF-36: The scale consists of social functioning, physical functioning, role limitations due to physical problems, mental health, role limitations due to emotional problems, energy/vitality, and perception of general health and pain subscales. The higher the score is the better the quality of life is $(11,12)$.

WEL Test: It was consisted of 20 questions about negative feelings, food accesibility, social pressure, physical disturbance and positive activity. The higher the score is the better the weight efficacy lifestyle is $(13,14)$.

HEl: It was developed by the United States Department of Agriculture to investigate the quality of diet in Americans(15). The new HEI-2015, which was published in 2018, consists of 13 components related to diet. The increase in scores indicates a positive development(16). Nutritional values for individuals based on their 24-hour food consumption records were calculated using the system) and measurements were calculated based on Dietary Guidelines for dietary data

RSES: The first 10 items of the scale are used to measure self-esteem. Self-esteem increases as the score obtained from the RSES decreases $(18,19)$.

HLSB-Il: The scale includes health responsibility, exercise, nutrition, self-actualization, interpersonal support, and stress management subscales $(20,21)$.

OWLQOL: The higher the score obtained from the scale is, the higher the quality of life of the person is(22, 23). 
MOtiVE Application for Mobile Phones: The program was developed by the researchers with taking technical support from a software engineer, specifically for this study and it is compatible with both Android and IOS. The messages were sent to the participants in the case group once a day for three months. Messages sent to the phone screen every morning at 9:00 AM were either text, visual or video messages. The visual messages designed to draw the attention of the reader included both a text and an illustration (Figure 2).

By sending text messages like "Sunday is the only day when most of us do not work. You will be more comfortable during the weekdays if you do your shopping and prepare meals on the weekend. Prepare your meatballs this Sunday and freeze them....", practical solutions were offered to the participants. By sending text messages like "Lifestyle changes improve your quality of life and enable you to lead a better quality of life. Improve your quality of life with diet and regular exercise. Thus, do not give up..." the participants were motivated.

Video messages were uploaded to YouTube and a link address (e.g.What is an antioxidant? https://www.youtube.com/watch?v=TlaQq1CbNBM) was sent to the cases, which enabled them to access. The videos were also subtitled so that the watcher could get the message in noisy environments. The registration screen of the MOtiVE, home screen layout, samples of messages sent for 90 days are given in Additional files.

One of the features of the MOtiVE is to calculate for how many days the participant was following the program and to send the message specific to that day to the person's phone. Thanks to this feature of the MOtiVE, it was possible to send a message like "Congratulations, you have been on a diet for a full month..." to the person who participated in the program for a month. So the program was made personspecific. Another feature of the MOtiVE is that only one-way communication was possible in order to avoid bias. The application enabled only the researchers to send messages to the participants but did not allow the participants to communicate with the researchers. In this way, standardization was established between the participants in terms of determining their knowledge and interest. Another feature of the MOtiVE is to report whether the participants see the message of that day, which enabled the researchers to find out whether the participant has read the messages.

\section{Statistical Analysis}

In the analysis of the study data, IBM SPSS Statistics (version 25; IBM, New York, NY, USA) was used. Continuous variables were presented with mean \pm standard deviation. At the baseline, the homogenously randomization of the participants to the case and control groups was investigated using the independent samples t-test (Mann Whitney-U test if parametric condition could not be met) and chi-square tests. The efficacy of the intervention was assessed using Wilcoxon signed rank test separately in the case and control groups. $p<0.05$ considered significant. 


\section{Ethical issues}

Study was conducted in accordance with the Declaration of Helsinki and ethical approval was obtained from University Medical Research Ethics Committee (no:17-7.1/14, 08.08.2017) and the written consent was obtained from the patients. The study was recorded in clinical trials (no: ClinicalTrials.gov NCT04026971).

\section{Results}

The number of the patients who took the first test was 79 (cases:39, controls:40). The number of individuals who took the final test was 38 (cases:20, controls:18). According to the feedback provided by MOtiVE, all participants read all the messages.

Of the participants, $90 \%$ in the case group and $83.4 \%$ in the control group were women. There was no significant age difference between cases and controls ( $34.7 \pm 14.0$ years vs. $38.7 \pm 13.5$ years; $p=0.349$ ). The analysis performed to test whether the participants who took the final test had been homogenously randomized to the case and control groups at the baseline revealed that there were no significant differences between the groups in terms of their sociodemographic characteristics, anthropometric measurements, or the scores they obtained from the RSES, OWLQOL, HLSB-II scale, WEL test and HEI and their sub-dimensions except for the nutrition subscale of the HLSB-II scale. The participants in the control group obtained significantly higher scores from the nutrition subscale of the HLSB-Il scale (Case group: 20.9 \pm 3.6 , Control group: $23.6 \pm 4.2, p=0.043$ ). Therefore, it can be said that the participants were homogenously distributed to the case and control groups.

\section{Anthropometric Measurements:}

Although weight, BMI and neck circumference measurements of the participants significantly decreased in both groups at the end of the 3 months, these differences were more dominant in the cases. Waist circumference decreased significantly only in the cases. The changes in the anthropometric measurements of the participants at the end of the three-month follow-up were given in Table 1. 
Table 1

Comparison of Anthropometric Measurements

\begin{tabular}{|c|c|c|c|c|c|c|c|c|c|}
\hline Measurements & Groups & $\mathrm{n}$ & & Mean & SD & Median & Min. & Max. & $p^{*}$ \\
\hline \multirow[t]{4}{*}{ Weight, kg } & \multirow[t]{2}{*}{ Case } & \multirow[t]{2}{*}{20} & $\begin{array}{l}\text { First } \\
\text { test }\end{array}$ & 91.4 & 18.0 & 89.3 & 67.7 & 129.0 & \multirow[t]{2}{*}{0.001} \\
\hline & & & $\begin{array}{l}\text { Final } \\
\text { test }\end{array}$ & 88.5 & 17.1 & 87.2 & 66.3 & 120.9 & \\
\hline & \multirow[t]{2}{*}{ Control } & \multirow[t]{2}{*}{18} & $\begin{array}{l}\text { First } \\
\text { test }\end{array}$ & 89.4 & 17.9 & 86.2 & 67.1 & 128.9 & \multirow[t]{2}{*}{0.006} \\
\hline & & & $\begin{array}{l}\text { Final } \\
\text { test }\end{array}$ & 86.4 & 16.9 & 83.7 & 61.8 & 125.4 & \\
\hline \multirow[t]{4}{*}{$\mathrm{BMI}, \mathrm{kg} / \mathrm{m}^{2}$} & \multirow[t]{2}{*}{ Case } & \multirow[t]{2}{*}{20} & $\begin{array}{l}\text { First } \\
\text { test }\end{array}$ & 33.8 & 6.0 & 33.2 & 25.7 & 45.7 & \multirow[t]{2}{*}{0.001} \\
\hline & & & $\begin{array}{l}\text { Final } \\
\text { test }\end{array}$ & 32.8 & 5.8 & 31.9 & 23.8 & 43.0 & \\
\hline & \multirow[t]{2}{*}{ Control } & \multirow[t]{2}{*}{18} & $\begin{array}{l}\text { First } \\
\text { test }\end{array}$ & 33.3 & 5.0 & 32.6 & 26.0 & 41.6 & \multirow[t]{2}{*}{0.006} \\
\hline & & & $\begin{array}{l}\text { Final } \\
\text { test }\end{array}$ & 32.2 & 4.7 & 32.5 & 23.8 & 40.4 & \\
\hline \multirow[t]{4}{*}{$\begin{array}{l}\text { Body Fat } \\
\text { Percentage, \% }\end{array}$} & \multirow[t]{2}{*}{ Case } & \multirow[t]{2}{*}{20} & $\begin{array}{l}\text { First } \\
\text { test }\end{array}$ & 39.5 & 5.2 & 26.6 & 46.7 & 40.3 & \multirow[t]{2}{*}{0.489} \\
\hline & & & $\begin{array}{l}\text { Final } \\
\text { test }\end{array}$ & 39.3 & 6.0 & 24.9 & 47.7 & 39.5 & \\
\hline & \multirow[t]{2}{*}{ Control } & \multirow[t]{2}{*}{18} & $\begin{array}{l}\text { First } \\
\text { test }\end{array}$ & 39.1 & 4.6 & 30.5 & 48.3 & 38.3 & \multirow[t]{2}{*}{0.556} \\
\hline & & & $\begin{array}{l}\text { Final } \\
\text { test }\end{array}$ & 38.7 & 4.9 & 29.3 & 46.0 & 39.1 & \\
\hline \multirow[t]{4}{*}{$\begin{array}{l}\text { Waist } \\
\text { circumference cm }\end{array}$} & \multirow[t]{2}{*}{ Case } & \multirow[t]{2}{*}{20} & $\begin{array}{l}\text { First } \\
\text { test }\end{array}$ & 108.2 & 15.1 & 86.0 & 141.0 & 109.5 & \multirow[t]{2}{*}{0.029} \\
\hline & & & $\begin{array}{l}\text { Final } \\
\text { test }\end{array}$ & 106.1 & 15.1 & 82.0 & 135.5 & 107.0 & \\
\hline & \multirow[t]{2}{*}{ Control } & \multirow[t]{2}{*}{18} & $\begin{array}{l}\text { First } \\
\text { test }\end{array}$ & 108.3 & 12.7 & 87.0 & 130.0 & 108.5 & \multirow[t]{2}{*}{0.060} \\
\hline & & & $\begin{array}{l}\text { Final } \\
\text { test }\end{array}$ & 105.5 & 12.4 & 86.0 & 126.0 & 105.0 & \\
\hline $\begin{array}{l}\text { Neck } \\
\text { circumference }(\mathrm{cm})\end{array}$ & Case & 20 & $\begin{array}{l}\text { First } \\
\text { test }\end{array}$ & 37.3 & 3.5 & 33.0 & 45.0 & 36.2 & 0.001 \\
\hline
\end{tabular}




\begin{tabular}{|c|c|c|c|c|c|c|c|c|c|}
\hline Measurements & Groups & $\mathrm{n}$ & & Mean & SD & Median & Min. & Max. & $\mathrm{p}^{*}$ \\
\hline & & & $\begin{array}{l}\text { Final } \\
\text { test }\end{array}$ & 36.4 & 3.3 & 33.0 & 44.5 & 35.7 & \\
\hline & Control & 18 & $\begin{array}{l}\text { First } \\
\text { test }\end{array}$ & 38.3 & 4.1 & 32.5 & 48.0 & 38.0 & 0.028 \\
\hline & & & $\begin{array}{l}\text { Final } \\
\text { test }\end{array}$ & 37.5 & 4.3 & 32.0 & 48.0 & 36.5 & \\
\hline
\end{tabular}

Scales: RSES scores significantly decreased in the cases which showed increase in self-esteem. There were not significant changes in WEL test scores of the participants in both groups. HLSB-II scale scores significantly increased only in the cases. As for the subscales of the HLSB-II scale, there were significant increases in the nutrition and stress management subscale scores in the cases. Except for the mental health subscale in the cases, the participants neither in the cases nor controls obtained significantly different mean scores from the QLSSF-36 and its subscales. While the mean HEl scores of the controls did not change significantly at the end of the three months, those of the cases increased significantly. The comparison of all the data collection tools used in the present study is presented in Table 2. 
Table 2

Comparison of all the data collection tools used in the present study

\begin{tabular}{|c|c|c|c|c|c|c|c|c|c|}
\hline Scales & Groups & $n$ & & Mean & SD & Median & Min. & Max. & $\mathrm{p}^{*}$ \\
\hline \multirow[t]{4}{*}{ RSES } & \multirow[t]{2}{*}{ Case } & \multirow[t]{2}{*}{20} & $\begin{array}{l}\text { First } \\
\text { test }\end{array}$ & 1.1 & 0.7 & 1.0 & 0.25 & 2.9 & \multirow[t]{2}{*}{0.035} \\
\hline & & & $\begin{array}{l}\text { Final } \\
\text { test }\end{array}$ & 0.7 & 0.5 & 0.6 & 0.25 & 2.0 & \\
\hline & \multirow[t]{2}{*}{ Control } & \multirow[t]{2}{*}{18} & $\begin{array}{l}\text { First } \\
\text { test }\end{array}$ & 0.9 & 0.5 & 0.8 & 0.25 & 2.3 & \multirow[t]{2}{*}{0.723} \\
\hline & & & $\begin{array}{l}\text { Final } \\
\text { test }\end{array}$ & 0.9 & 0.6 & 0.7 & 0 & 2.3 & \\
\hline \multirow[t]{4}{*}{ OWLQOL } & \multirow[t]{2}{*}{ Case } & \multirow[t]{2}{*}{20} & $\begin{array}{l}\text { First } \\
\text { test }\end{array}$ & 55.2 & 16.6 & 58.0 & 28.0 & 87.0 & \multirow[t]{2}{*}{0.064} \\
\hline & & & $\begin{array}{l}\text { Final } \\
\text { test }\end{array}$ & 45.3 & 21.5 & 38.5 & 20.0 & 95.0 & \\
\hline & \multirow[t]{2}{*}{ Control } & \multirow[t]{2}{*}{18} & $\begin{array}{l}\text { First } \\
\text { test }\end{array}$ & 51.3 & 21.0 & 50.0 & 11.0 & 95.0 & \multirow[t]{2}{*}{0.331} \\
\hline & & & $\begin{array}{l}\text { Final } \\
\text { test }\end{array}$ & 49.7 & 22.4 & 52.5 & 2.0 & 93.0 & \\
\hline \multirow[t]{4}{*}{ HLSB-II } & \multirow[t]{2}{*}{ Case } & \multirow[t]{2}{*}{20} & $\begin{array}{l}\text { First } \\
\text { test }\end{array}$ & 131.0 & 15.4 & 126.5 & 11.0 & 173.0 & \multirow[t]{2}{*}{0.021} \\
\hline & & & $\begin{array}{l}\text { Final } \\
\text { test }\end{array}$ & 141.0 & 19.5 & 144.0 & 111.0 & 188.0 & \\
\hline & \multirow[t]{2}{*}{ Control } & \multirow[t]{2}{*}{18} & $\begin{array}{l}\text { First } \\
\text { test }\end{array}$ & 135.0 & 20.2 & 135.0 & 99.0 & 177.0 & \multirow[t]{2}{*}{0.981} \\
\hline & & & $\begin{array}{l}\text { Final } \\
\text { test }\end{array}$ & 135.0 & 19.3 & 129.5 & 109.0 & 167.0 & \\
\hline \multirow[t]{4}{*}{ WEL Test } & \multirow[t]{2}{*}{ Case } & \multirow[t]{2}{*}{20} & $\begin{array}{l}\text { First } \\
\text { test }\end{array}$ & 51.0 & 13.7 & 48.0 & 24.0 & 89.0 & \multirow[t]{2}{*}{0.256} \\
\hline & & & $\begin{array}{l}\text { Final } \\
\text { test }\end{array}$ & 47.7 & 16.9 & 46.5 & 20.0 & 91.0 & \\
\hline & \multirow[t]{2}{*}{ Control } & \multirow[t]{2}{*}{18} & $\begin{array}{l}\text { First } \\
\text { test }\end{array}$ & 47.2 & 16.8 & 48 & 20.0 & 83.0 & \multirow[t]{2}{*}{0.177} \\
\hline & & & $\begin{array}{l}\text { Final } \\
\text { test }\end{array}$ & 42.0 & 12.7 & 39.0 & 26.0 & 70.0 & \\
\hline $\begin{array}{l}\text { QLSSF-36 } \\
\text { Physical Function }\end{array}$ & Case & 20 & $\begin{array}{l}\text { First } \\
\text { test }\end{array}$ & 74.7 & 24.6 & 77.5 & 25.0 & 100.0 & 0.138 \\
\hline
\end{tabular}




\begin{tabular}{|c|c|c|c|c|c|c|c|c|c|}
\hline Scales & Groups & $\mathrm{n}$ & & Mean & SD & Median & Min. & Max. & $\mathrm{p}^{*}$ \\
\hline & & & $\begin{array}{l}\text { Final } \\
\text { test }\end{array}$ & 80.5 & 18.5 & 90.0 & 45.0 & 100.0 & \\
\hline & Control & 18 & $\begin{array}{l}\text { First } \\
\text { test }\end{array}$ & 67.2 & 30.0 & 70.0 & 0 & 100.0 & 0.506 \\
\hline & & & $\begin{array}{l}\text { Final } \\
\text { test }\end{array}$ & 72.5 & 31.9 & 82.5 & 0 & 100.0 & \\
\hline \multirow{4}{*}{$\begin{array}{l}\text { QLSSF-36 } \\
\text { Role Limitations Due } \\
\text { to Physical Problems }\end{array}$} & Case & 20 & $\begin{array}{l}\text { First } \\
\text { test }\end{array}$ & 70.0 & 39.4 & 100.0 & 0 & 100.0 & \multirow[t]{2}{*}{0.783} \\
\hline & & & $\begin{array}{l}\text { Final } \\
\text { test }\end{array}$ & 68.7 & 45.0 & 100.0 & 0 & 100.0 & \\
\hline & Control & 18 & $\begin{array}{l}\text { First } \\
\text { test }\end{array}$ & 56.9 & 43.5 & 75.0 & 0 & 100.0 & \multirow[t]{2}{*}{0.363} \\
\hline & & & $\begin{array}{l}\text { Final } \\
\text { test }\end{array}$ & 68.0 & 40.9 & 100.0 & 0 & 100.0 & \\
\hline \multirow{4}{*}{$\begin{array}{l}\text { QLSSF-36 } \\
\text { Role Limitations Due } \\
\text { to Emotional } \\
\text { Problems }\end{array}$} & Case & 20 & $\begin{array}{l}\text { First } \\
\text { test }\end{array}$ & 70.0 & 38.8 & 100.0 & 0 & 100.0 & \multirow[t]{2}{*}{0.181} \\
\hline & & & $\begin{array}{l}\text { Final } \\
\text { test }\end{array}$ & 56.6 & 42.0 & 66.6 & 0 & 100.0 & \\
\hline & Control & 18 & $\begin{array}{l}\text { First } \\
\text { test }\end{array}$ & 55.5 & 47.1 & 66.6 & 0 & 100.0 & \multirow[t]{2}{*}{0.786} \\
\hline & & & $\begin{array}{l}\text { Final } \\
\text { test }\end{array}$ & 51.8 & 47.4 & 50.0 & 0 & 100.0 & \\
\hline \multirow[t]{4}{*}{$\begin{array}{l}\text { QLSSF-36 } \\
\text { Energy / Vitality }\end{array}$} & Case & 20 & $\begin{array}{l}\text { First } \\
\text { test }\end{array}$ & 51.0 & 15.4 & 50.0 & 20.0 & 75.0 & \multirow[t]{2}{*}{0.203} \\
\hline & & & $\begin{array}{l}\text { Final } \\
\text { test }\end{array}$ & 58.2 & 16.1 & 60.0 & 25.0 & 85.0 & \\
\hline & Control & 18 & $\begin{array}{l}\text { First } \\
\text { test }\end{array}$ & 50.2 & 20.8 & 52.5 & 10.0 & 80.0 & \multirow[t]{2}{*}{0.376} \\
\hline & & & $\begin{array}{l}\text { Final } \\
\text { test }\end{array}$ & 53.6 & 19.5 & 55.0 & 0 & 95.0 & \\
\hline \multirow[t]{3}{*}{$\begin{array}{l}\text { QLSSF-36 } \\
\text { Mental Health }\end{array}$} & Case & 20 & $\begin{array}{l}\text { First } \\
\text { test }\end{array}$ & 57.6 & 16.6 & 60.0 & 16.0 & 80.0 & \multirow[t]{2}{*}{0.048} \\
\hline & & & $\begin{array}{l}\text { Final } \\
\text { test }\end{array}$ & 67.4 & 12.3 & 70.0 & 32.0 & 80.0 & \\
\hline & Control & 18 & $\begin{array}{l}\text { First } \\
\text { test }\end{array}$ & 57.1 & 21.7 & 54.0 & 8.0 & 96.0 & 0.583 \\
\hline
\end{tabular}




\begin{tabular}{|c|c|c|c|c|c|c|c|c|c|}
\hline Scales & Groups & $\mathbf{n}$ & & Mean & SD & Median & Min. & Max. & $\mathrm{p}^{*}$ \\
\hline & & & $\begin{array}{l}\text { Final } \\
\text { test }\end{array}$ & 58.0 & 19.3 & 56.0 & 4.0 & 100.0 & \\
\hline \multirow[t]{4}{*}{$\begin{array}{l}\text { QLSSF-36 } \\
\text { Social Function }\end{array}$} & \multirow[t]{2}{*}{ Case } & \multirow[t]{2}{*}{20} & $\begin{array}{l}\text { First } \\
\text { test }\end{array}$ & 69.3 & 25.1 & 62.5 & 25.0 & 100.0 & \multirow[t]{2}{*}{0.526} \\
\hline & & & $\begin{array}{l}\text { Final } \\
\text { test }\end{array}$ & 75.6 & 26.4 & 87.5 & 25.0 & 100.0 & \\
\hline & \multirow[t]{2}{*}{ Control } & \multirow[t]{2}{*}{18} & $\begin{array}{l}\text { First } \\
\text { test }\end{array}$ & 60.4 & 30.6 & 62.5 & 0 & 100.0 & \multirow[t]{2}{*}{0.104} \\
\hline & & & $\begin{array}{l}\text { Final } \\
\text { test }\end{array}$ & 75.0 & 21.8 & 75.0 & 37.5 & 100.0 & \\
\hline \multirow[t]{4}{*}{$\begin{array}{l}\text { QLSSF-36 } \\
\text { Pain }\end{array}$} & \multirow[t]{2}{*}{ Case } & \multirow[t]{2}{*}{20} & $\begin{array}{l}\text { First } \\
\text { test }\end{array}$ & 68.7 & 25.6 & 67.5 & 10.0 & 100.0 & \multirow[t]{2}{*}{0.811} \\
\hline & & & $\begin{array}{l}\text { Final } \\
\text { test }\end{array}$ & 70.3 & 25.3 & 77.5 & 32.5 & 100.0 & \\
\hline & \multirow[t]{2}{*}{ Control } & \multirow[t]{2}{*}{18} & $\begin{array}{l}\text { First } \\
\text { test }\end{array}$ & 69.3 & 28.9 & 67.5 & 10.0 & 100.0 & \multirow[t]{2}{*}{0.218} \\
\hline & & & $\begin{array}{l}\text { Final } \\
\text { test }\end{array}$ & 75.5 & 22.8 & 85.0 & 22.5 & 100.0 & \\
\hline \multirow{4}{*}{$\begin{array}{l}\text { QLSSF-36 } \\
\text { Perception of } \\
\text { General Health }\end{array}$} & \multirow[t]{2}{*}{ Case } & \multirow[t]{2}{*}{20} & $\begin{array}{l}\text { First } \\
\text { test }\end{array}$ & 51.0 & 21.2 & 50.0 & 15.0 & 90.0 & \multirow[t]{2}{*}{0.982} \\
\hline & & & $\begin{array}{l}\text { Final } \\
\text { test }\end{array}$ & 53.2 & 19.4 & 57.5 & 10.0 & 80.0 & \\
\hline & \multirow[t]{2}{*}{ Control } & \multirow[t]{2}{*}{18} & $\begin{array}{l}\text { First } \\
\text { test }\end{array}$ & 48.6 & 18.2 & 47.5 & 20.0 & 80.0 & \multirow[t]{2}{*}{0.206} \\
\hline & & & $\begin{array}{l}\text { Final } \\
\text { test }\end{array}$ & 53.8 & 20.4 & 55.0 & 5.0 & 85.0 & \\
\hline \multirow[t]{4}{*}{$\begin{array}{l}\text { HLSB-II Health } \\
\text { Responsibility }\end{array}$} & \multirow[t]{2}{*}{ Case } & \multirow[t]{2}{*}{20} & $\begin{array}{l}\text { First } \\
\text { test }\end{array}$ & 21.7 & 4.7 & 21.0 & 12.0 & 31.0 & \multirow[t]{2}{*}{0.294} \\
\hline & & & $\begin{array}{l}\text { Final } \\
\text { test }\end{array}$ & 23.0 & 5.2 & 23.0 & 16.0 & 33.0 & \\
\hline & \multirow[t]{2}{*}{ Control } & \multirow[t]{2}{*}{18} & $\begin{array}{l}\text { First } \\
\text { test }\end{array}$ & 22.8 & 5.4 & 23.0 & 11.0 & 33.0 & \multirow[t]{2}{*}{0.896} \\
\hline & & & $\begin{array}{l}\text { Final } \\
\text { test }\end{array}$ & 22.8 & 5.3 & 22.0 & 14.0 & 33.0 & \\
\hline HLSB-II Exercise & Case & 20 & $\begin{array}{l}\text { First } \\
\text { test }\end{array}$ & 15.7 & 4.2 & 15.5 & 8.0 & 24.0 & 0.245 \\
\hline
\end{tabular}




\begin{tabular}{|c|c|c|c|c|c|c|c|c|c|}
\hline \multirow[t]{4}{*}{ Scales } & \multirow[t]{2}{*}{ Groups } & \multirow[t]{2}{*}{$\mathrm{n}$} & & Mean & SD & Median & Min. & Max. & $\mathrm{p}^{*}$ \\
\hline & & & $\begin{array}{l}\text { Final } \\
\text { test }\end{array}$ & 17.0 & 5.6 & 17.5 & 8.0 & 29.0 & \\
\hline & \multirow[t]{2}{*}{ Control } & \multirow[t]{2}{*}{18} & $\begin{array}{l}\text { First } \\
\text { test }\end{array}$ & 14.8 & 5.4 & 14.0 & 8.0 & 25.0 & \multirow[t]{2}{*}{0.325} \\
\hline & & & $\begin{array}{l}\text { Final } \\
\text { test }\end{array}$ & 16.1 & 4.6 & 16.5 & 9.0 & 24.0 & \\
\hline \multirow[t]{4}{*}{ HLSB-II Nutrition } & \multirow[t]{2}{*}{ Case } & \multirow[t]{2}{*}{20} & $\begin{array}{l}\text { First } \\
\text { test }\end{array}$ & 20.9 & 3.6 & 21.0 & 15.0 & 31.0 & \multirow[t]{2}{*}{0.001} \\
\hline & & & $\begin{array}{l}\text { Final } \\
\text { test }\end{array}$ & 25.0 & 4.2 & 25.5 & 17.0 & 36.0 & \\
\hline & \multirow[t]{2}{*}{ Control } & \multirow[t]{2}{*}{18} & $\begin{array}{l}\text { First } \\
\text { test }\end{array}$ & 23.6 & 4.2 & 23.5 & 18.0 & 33.0 & \multirow[t]{2}{*}{0.950} \\
\hline & & & $\begin{array}{l}\text { Final } \\
\text { test }\end{array}$ & 23.9 & 4.1 & 23.0 & 18.0 & 32.0 & \\
\hline \multirow[t]{4}{*}{$\begin{array}{l}\text { HLSB-II } \\
\text { Self-actualization }\end{array}$} & \multirow[t]{2}{*}{ Case } & \multirow[t]{2}{*}{20} & $\begin{array}{l}\text { First } \\
\text { test }\end{array}$ & 26.7 & 2.9 & 26.5 & 21.0 & 33.0 & \multirow[t]{2}{*}{0.124} \\
\hline & & & $\begin{array}{l}\text { Final } \\
\text { test }\end{array}$ & 27.7 & 3.1 & 28.0 & 20.0 & 34.0 & \\
\hline & \multirow[t]{2}{*}{ Control } & \multirow[t]{2}{*}{18} & $\begin{array}{l}\text { First } \\
\text { test }\end{array}$ & 26.8 & 3.8 & 26.0 & 21.0 & 34.0 & \multirow[t]{2}{*}{0.457} \\
\hline & & & $\begin{array}{l}\text { Final } \\
\text { test }\end{array}$ & 26.6 & 4.2 & 26.0 & 19.0 & 34.0 & \\
\hline \multirow[t]{4}{*}{$\begin{array}{l}\text { HLSB-II Interpersonal } \\
\text { Support }\end{array}$} & \multirow[t]{2}{*}{ Case } & \multirow[t]{2}{*}{20} & $\begin{array}{l}\text { First } \\
\text { test }\end{array}$ & 26.9 & 3.5 & 27.5 & 21.0 & 33.0 & \multirow[t]{2}{*}{0.323} \\
\hline & & & $\begin{array}{l}\text { Final } \\
\text { test }\end{array}$ & 27.5 & 4.1 & 29.0 & 21.0 & 35.0 & \\
\hline & \multirow[t]{2}{*}{ Control } & \multirow[t]{2}{*}{18} & $\begin{array}{l}\text { First } \\
\text { test }\end{array}$ & 27.7 & 4.1 & 28.0 & 21.0 & 36.0 & \multirow[t]{2}{*}{0.175} \\
\hline & & & $\begin{array}{l}\text { Final } \\
\text { test }\end{array}$ & 26.3 & 4.6 & 24.5 & 18.0 & 35.0 & \\
\hline \multirow[t]{3}{*}{$\begin{array}{l}\text { HLSB-II Stress } \\
\text { Management }\end{array}$} & \multirow[t]{2}{*}{ Case } & \multirow[t]{2}{*}{20} & $\begin{array}{l}\text { First } \\
\text { test }\end{array}$ & 18.9 & 2.9 & 18.5 & 15.0 & 25.0 & \multirow[t]{2}{*}{0.036} \\
\hline & & & $\begin{array}{l}\text { Final } \\
\text { test }\end{array}$ & 20.7 & 3.2 & 21.0 & 14.0 & 28.0 & \\
\hline & Control & 18 & $\begin{array}{l}\text { First } \\
\text { test }\end{array}$ & 19.0 & 3.3 & 19.5 & 13.0 & 26.0 & 0.876 \\
\hline
\end{tabular}




\begin{tabular}{|c|c|c|c|c|c|c|c|c|c|}
\hline Scales & Groups & $n$ & & Mean & SD & Median & Min. & Max. & $p^{*}$ \\
\hline & & & $\begin{array}{l}\text { Final } \\
\text { test }\end{array}$ & 19.1 & 3.1 & 18.0 & 14.0 & 26.0 & \\
\hline \multirow[t]{4}{*}{ HEI Score } & Case & 20 & $\begin{array}{l}\text { First } \\
\text { test }\end{array}$ & 49.2 & 9.8 & 52.0 & 30.0 & 65.0 & 0.007 \\
\hline & & & $\begin{array}{l}\text { Final } \\
\text { test }\end{array}$ & 56.1 & 11.4 & 56.5 & 34.0 & 72.0 & \\
\hline & Control & 18 & $\begin{array}{l}\text { First } \\
\text { test }\end{array}$ & 53.7 & 8.2 & 54.0 & 43.0 & 68.5 & 0.486 \\
\hline & & & $\begin{array}{l}\text { Final } \\
\text { test }\end{array}$ & 55.7 & 10.3 & 55.7 & 37.0 & 72.5 & \\
\hline
\end{tabular}

\section{Discussion}

The use of internet-based applications to develop a healthy lifestyle is increasing. However, most of these programs have not been evaluated with appropriate and standardized methods and have different exposure times. Saffron Naimark et al. investigated the effect of an internet-based application on improving healthy lifestyle in a randomized controlled trial. They collected data on nutrition knowledge, diet quality and physical activity periods using online data collection forms. The cases used the Internetbased application designed based on healthy lifestyle recommendations of the US Department of Agriculture and the Israeli Ministry of Health for 14 weeks. Of the 99 participants $86 \%$ of them $(n=85,56$ in case group, 29 in control group) completed the study. Besides significant weight loss ( $p=0.03)$, knowledge score, diet quality score, and success score indicating success in maintaining a healthy life increased significantly in the cases. There was a significant correlation between the frequency of using the application and high success score $(p<0.01)(24)$. Similarly in their randomized controlled study aimed at weight loss, Kevin Patrick et al. sent SMSs and/or multimedia messages to the participants in the case group 2-5 times a day for 16 weeks. At the end of the $16^{\text {th }}$ week the weight loss in the cases was significantly higher $(p=0.02)$. It was concluded that SMSs and multimedia messages could promote behaviors supporting weight loss in obese adults(25). In present study, all participants took nutritional trainig first with their personal diets and then randomized to cases and controls. That could be the reason for controls to loose weight significantly as well as cases, cases being more prodominant. Besides weight loss, present study showed significant improvements regarding self-esteem; healthy lifestyle behaviors, quality of life and healthy eating habits were observed only cases. Therefore, in line with Kevin Patrick et al.'s study, mobile applications can improve behaviors that support weight loss.

In a meta-analysis including 14 randomized controlled trials to investigate whether internet-based interventions were effective in empowering patients concluded that these interventions yield to positive improvements. On the other hand, in 3 studies using general self-efficacy scales and in 1 study using the RSES conducted to assess self-esteem, no changes were observed. The comparison of face-to-face 
interviews and internet-based interventions demonstrated that no significant differences were observed in self-esteem(26). In the present study, cases received messages addition to the routine nutritional therapy. Unlike the meta-analysis, a significant decrease in RSES scores thus, a significant increase in self-esteem was observed in cases in the present study. However, according to the analysis of the self-efficacy scores obtained from the WEL test, we also could not show significant changes in both groups. It is thought that internet-based interventions can be used to improve self-esteem, lifestyle behaviors and quality of life of patients but not self-efficacy.

Although the use of a mobile application led to changes in health behaviors, the mechanisms by which these applications facilitate behavior change are generally not known. Joshua $\mathrm{H}$ West et al. conducted a cross-sectional study including 217 participants. The participants gave their feedback about their diet and nutrition applications in the last 6 months and most of the participants agreed that the application increased their dietary motivation, improved their self-efficacy, and increased their willingness to set dietary goals and to reach the target. Therefore, it was concluded that diet and nutrition-related practices focusing on the improvement of motivation, willingness, self-efficacy, attitude, knowledge and goal setting might be particularly useful(27). Jacobs et al demonstrated the importance of adherence to intervention on weight loss in a large sample using a smartphone application Noom tracking individual self-monitoring and showed that after three months, significant reduction in BMI was accomplished. They concluded that smart phone application use can induce weight loss associated with adherence(28). The present study was also aimed at improving healthy lifestyle behaviors via inducing motivation. At the end of the present study, there was a significant improvement in the mean scores BMI as well as from the overall HLSB-II scale and only its nutrition and stress management subscales.

\section{Limitations and Strengths}

The mobile application MOtiVE and its unique features solely designed for this study can be considered as the main strenght to this study. Personel feedback about messages, personel timing and sending messages according to enrollment time makes this programe tailored to participant. Moreover, one-way communication ensured standardization about knowledge and motivation. The main limitation of this study was losses during follow-up period. Nearly half of the participants didn't complete the study. In cases, even though they fallow the messages, some of them refused to come for post-test and final measurements. This may be due to conducting this study in a university hospital where control appoinments could only be given for three months later. This showed us that people need stronger motivations to continue follow-ups.

\section{Conclusions}

In conclusion, it is thought that provision of mobile application based nutrition education to overweight and obese individuals in addition to the routine nutritional therapy may lead to improvements in anthropometric measurements, self-esteem, healthy lifestyle behaviors, quality of life and healthy eating habits of the participants, and it might help to achieve the targeted weight loss. As the number of dietary 
and nutritional practices continues to increase, to ensure healthy lifestyle behavior changes, application developers together with health professional should consider integrating the appropriate theoretical structures into the newly developed mobile applications Because these types of mobile applications are easily accessible, such applications should be created and made available to the public free of charge in the protection of public health, and prevention and reduction of obesity.

\section{List Of Abbreviations:}

Quality of Life (QoL)

Body Mass Index (BMI)

Weight Efficacy Lifestyle (WEL) test,

Quality of Life Scale SF-36 (QoLSSF-36),

Rosenberg Self-Esteem Scale (RSES),

Obesity and Weight-Loss Quality of Life Instrument (OWLQoL),

Healthy Lifestyle Behavior-II Scale (HLSB-II)

Healthy Eating Index (HEI)

\section{Declarations}

\section{CRediT authorship contribution statement}

All the authors declare that, according to CRediT (Contributor Roles Taxonomy), they had substantial contributions to the conception or design of the work as;

DP: Conceptualization, Methodology, Software, Investigation, Formal analysis, Writing-original draft, reviewing and editing, Supervision, Funding acquisition

SS: Conceptualization, Methodology, Investigation, Writing- reviewing and editing, Supervision.

RM: Conceptualization, Methodology, Software, Formal analysis, Writing-original draft, reviewing and editing, Supervision, Funding acquisition

All the authors declare that they approved the final version to be published.

\section{Ethical issues}

Study was conducted in accordance with the Declaration of Helsinki and ethical approval was obtained from University Medical Research Ethics Committee (no:17-7.1/14, 08.08.2017) and the written 
consent was obtained from the patients. The study was recorded in clinical trials (no: ClinicalTrials.gov NCT04026971).

\section{Availability of data and materials}

The datasets used and/or analyzed during the current study are available from the corresponding author on reasonable request.

Acknowledgments: We would like to extend our sincere thanks to the University Scientific Research Projects Coordination Bureau due to their invaluable support to the research (No: 17-SBE-004).

Funding: This study was funded by University Scientific Research Projects Coordination Bureau (No: 17-SBE-004). The bureau had no effect on study design, the collection, analysis, and interpretation of data; in the writing of the report; and in the decision to submit the article for publication.

\section{Conflicts of Interest: None}

\section{References}

1. Gómez-Donoso C, Martínez-González MA, Gea A, Murphy KJ, Parletta N, Bes-Rastrollo M. A foodbased score and incidence of overweight/obesity: The Dietary Obesity-Prevention Score (DOS). Clinical Nutrition. 2019;38(6):2607-15.

2. Watson S, Woodside JV, Ware LJ, Hunter SJ, McGrath A, Cardwell CR, et al. Effect of a Web-Based Behavior Change Program on Weight Loss and Cardiovascular Risk Factors in Overweight and Obese Adults at High Risk of Developing Cardiovascular Disease: Randomized Controlled Trial. J Med Internet Res. 2015;17(7):e177.

3. Block G, Azar KM, Romanelli RJ, Block TJ, Hopkins D, Carpenter HA, et al. Diabetes Prevention and Weight Loss with a Fully Automated Behavioral Intervention by Email, Web, and Mobile Phone: A Randomized Controlled Trial Among Persons with Prediabetes. J Med Internet Res. 2015;17(10):e240.

4. Flores Mateo G, Granado-Font E, Ferre-Grau C, Montana-Carreras X. Mobile Phone Apps to Promote Weight Loss and Increase Physical Activity: A Systematic Review and Meta-Analysis. J Med Internet Res. 2015;17(11):e253.

5. DiFilippo KN, Huang W-HD, Chapman-Novakofski KM. Mobile Apps for the Dietary Approaches to Stop Hypertension (DASH): App Quality Evaluation. Journal of Nutrition Education and Behavior. 2018;50(6):620-5.

6. Breines J, Toole A, Tu C, Chen S. Self-compassion, Body Image, and Self-reported Disordered Eating. Self and Identity. 2014;13(4):432-48.

7. Kelly AC, Vimalakanthan K, Carter JC. Understanding the roles of self-esteem, self-compassion, and fear of self-compassion in eating disorder pathology: An examination of female students and eating disorder patients. Eating Behaviors. 2014;15(3):388-91. 
8. Aguilar-Martínez A, Sole-Sedeno JM, Mancebo-Moreno G, Medina FX, Carreras-Collado R, Saigí-Rubió F. Use of mobile phones as a tool for weight loss: a systematic review. Journal of telemedicine and telecare. 2014;20(6):339-49.

9. Allen JK, Stephens J, Dennison Himmelfarb CR, Stewart KJ, Hauck S. Randomized controlled pilot study testing use of smartphone technology for obesity treatment. Journal of obesity. 2013;2013.

10. Meseri R, Ucku R, Unal B. Waist:height ratio: a superior index in estimating cardiovascular risks in Turkish adults. Public Health Nutr. 2014;17(10):2246-52.

11. Ware Jr JE, Sherbourne CD. The MOS 36-item short-form health survey (SF-36): I. Conceptual framework and item selection. Medical care. 1992:473-83.

12. Kocyigit $\mathrm{H}$. Reliability and validity of the Turkish version of short form-36 (SF-36): a study in a group of patients will rheumatic diseases. Turk J Drugs Ther. 1999;12:102-6.

13. Clark MM, Abrams DB, Niaura RS, Eaton CA, Rossi JS. Self-efficacy in weight management. Journal of consulting and clinical psychology. 1991;59(5):739.

14. Bozan N, Bas, M., Cigerim, N. Relationship between eating self-efficacy and abnormal eating behaviors among adolescent girls. V International Nutrition and Dietetic Congress. 2016.

15. T Kennedy E, Ohls J, Carlson S, Fleming K. The healthy eating index: design and applications. Journal of the American Dietetic Association. 1995;95(10):1103-8.

16. Krebs-Smith SM, Pannucci TE, Subar AF, Kirkpatrick SI, Lerman JL, Tooze JA, et al. Update of the healthy eating index: HEI-2015. Journal of the Academy of Nutrition and Dietetics. 2018;118(9):1591602.

17. Nutritional Guideline in Turkey, 2015 Ankara: Ministry of Health, Public Health Directorate; 2016. Report No.: 1031.

18. Rosenberg M. Rosenberg self-esteem scale (RSE). Acceptance and commitment therapy Measures package. 1965;61:52.

19. Sevincer GM, Kaya A, Bozkurt S, Akin E, Kose S. Reliability, validity, and factorial structure of the Turkish version of the Weight Self-Stigma Questionnaire (Turkish WSSQ). Psychiatry and Clinical Psychopharmacology. 2017;27(4):386-92.

20. Walker S, Hill-Polerecky D. Psychometric evaluation of the health-promoting lifestyle profile II. Unpublished manuscript, University of Nebraska Medical Center. 1996:120-26.

21. Bahar Z, Beser A, Gordes N, Ersin F, KIssal A. Healthy life style behavior scale II: A reliability and validity study. Journal of Cumhuriyet University School of Nursing. 2008;12(1):1-13.

22. Patrick DL, Bushnell DM, Rothman M. Performance of two self-report measures for evaluating obesity and weight loss. Obesity research. 2004;12(1):48-57.

23. Gündüzoğlu NÇ, Fadıloğlu Ç, Yılmaz C. The examination of validity and reliability for obese individuals specific quality of life scale. Anatolian Journal of Psychiatry. 2014;15(1):63-8.

24. Safran Naimark J, Madar Z, R Shahar D. The Impact of a Web-Based App (eBalance) in Promoting Healthy Lifestyles: Randomized Controlled Trial. J Med Internet Res. 2015;17(3):e56. 
25. Patrick K, Raab F, Adams MA, Dillon L, Zabinski M, Rock CL, et al. A Text Message-Based Intervention for Weight Loss: Randomized Controlled Trial. J Med Internet Res. 2009;11(1):e1.

26. Samoocha D, Bruinvels DJ, Elbers NA, Anema JR, van der Beek AJ. Effectiveness of Web-based Interventions on Patient Empowerment: A Systematic Review and Meta-analysis. J Med Internet Res. 2010;12(2):e23.

27. West JH, Belvedere LM, Andreasen R, Frandsen C, Hall PC, Crookston BT. Controlling Your "App"etite: How Diet and Nutrition-Related Mobile Apps Lead to Behavior Change. JMIR Mhealth Uhealth. 2017;5(7):e95.

28. Jacobs S, Radnitz C, Hildebrandt T. Adherence as a predictor of weight loss in a commonly used smartphone application. Obesity research \& clinical practice. 2017;11(2):206-14.

\section{Figures}

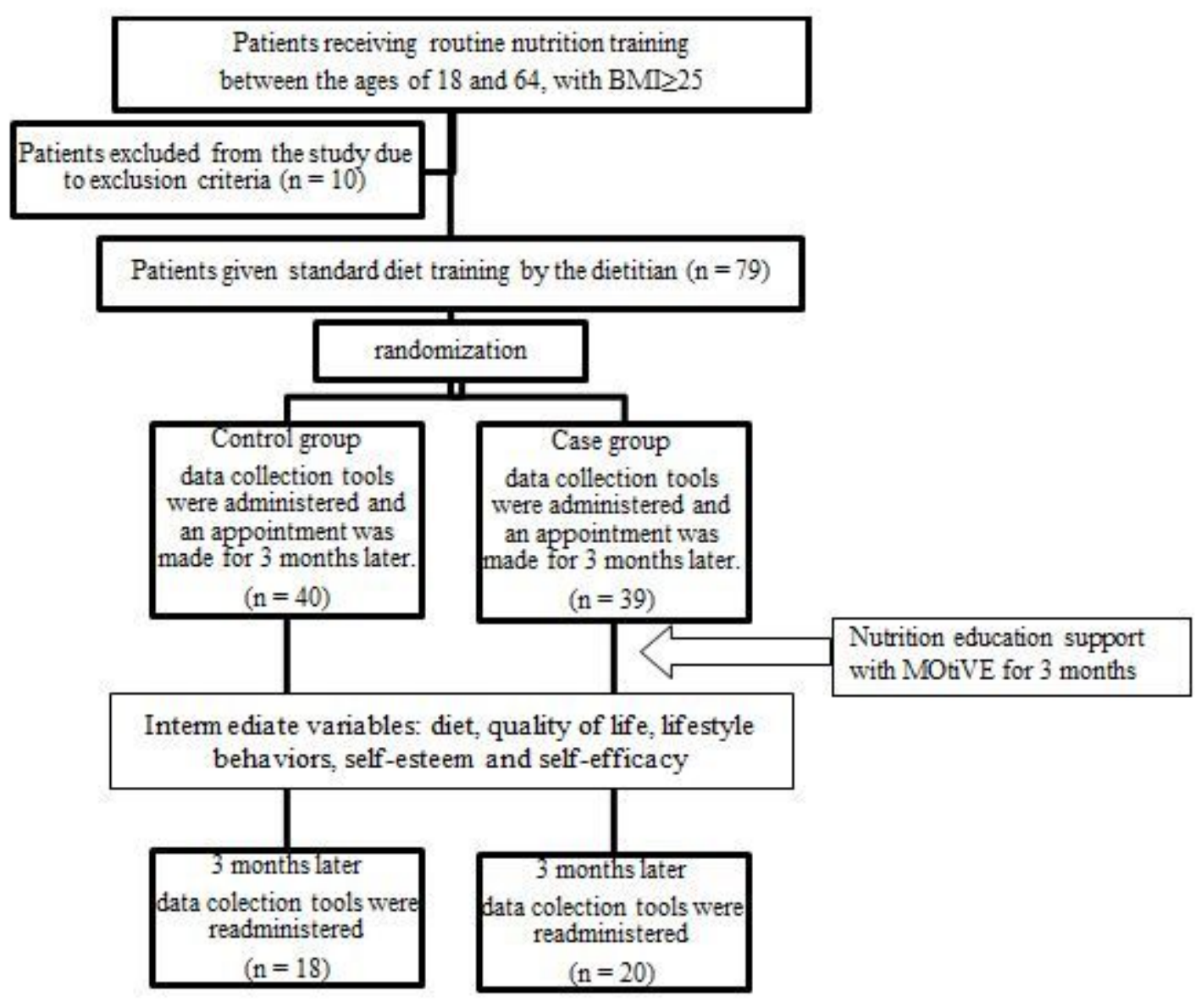

\section{Figure 1}

The flowchart of the study 

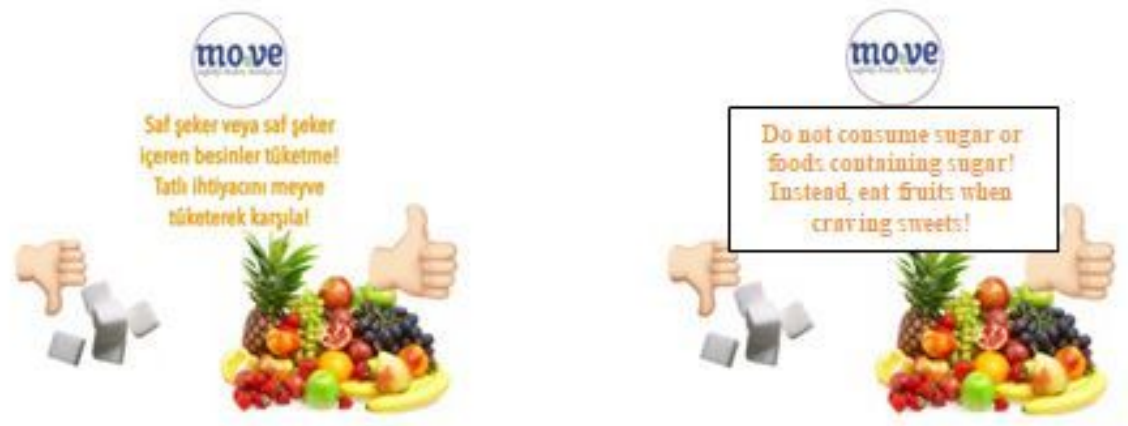

Figure 2

Visual Message Example

\section{Supplementary Files}

This is a list of supplementary files associated with this preprint. Click to download.

- Additionalfiles.docx 